Review Article

\title{
Pressor Response to Noradrenaline in the Setting of Septic Shock: Anything New under the Sun-Dexmedetomidine, Clonidine? A Minireview
}

\author{
A. Géloën, ${ }^{1}$ C. Pichot, ${ }^{2}$ S. Leroy, ${ }^{3}$ C. Julien, ${ }^{2}$ M. Ghignone, ${ }^{2}$ C. N. May, ${ }^{4}$ and L. Quintin ${ }^{2}$ \\ ${ }^{1}$ CARMeN, INSERM U 1060, Insa de Lyon, Villeurbanne, France \\ ${ }^{2}$ Physiology (EA 4612: "Neurocardiology"), University of Lyon, 69622 Lyon, France \\ ${ }^{3} U M I$ 233, Institut de Recherche du Developpement, Montpellier, France \\ ${ }^{4}$ Florey Institute of Neuroscience and Mental Health, Parkville, VIC 3052, Australia \\ Correspondence should be addressed to L. Quintin; lucquintin@yahoo.com
}

Received 20 July 2015; Accepted 30 August 2015

Academic Editor: Zsolt Molnar

Copyright (C) 2015 A. Géloën et al. This is an open access article distributed under the Creative Commons Attribution License, which permits unrestricted use, distribution, and reproduction in any medium, provided the original work is properly cited.

Progress over the last 50 years has led to a decline in mortality from $\approx 70 \%$ to $\approx 20 \%$ in the best series of patients with septic shock. Nevertheless, refractory septic shock still carries a mortality close to $100 \%$. In the best series, the mortality appears related to multiple organ failure linked to comorbidities and/or an intense inflammatory response: shortening the period that the subject is exposed to circulatory instability may further lower mortality. Treatment aims at reestablishing circulation within a "central" compartment (i.e., brain, heart, and lung) but fails to reestablish a disorganized microcirculation or an adequate response to noradrenaline, the most widely used vasopressor. Indeed, steroids, nitric oxide synthase inhibitors, or donors have not achieved overwhelming acceptance in the setting of septic shock. Counterintuitively, $\alpha_{2}$-adrenoceptor agonists were shown to reduce noradrenaline requirements in two cases of human septic shock. This has been replicated in rat and sheep models of sepsis. In addition, some data show that $\alpha_{2}$-adrenoceptor agonists lead to an improvement in the microcirculation. Evidence-based documentation of the effects of alpha-2 agonists is needed in the setting of human septic shock.

\section{Introduction}

Following immediate resuscitation [1], the clinician treating septic shock faces different issues including (a) recoupling the peripheral compartment (i.e., the microcirculation) to the "central" compartment (i.e., brain, heart, and lung) and (b) restoring the pressor response to vasopressors, usually noradrenaline (NA). This minireview addresses these issues in the setting of septic shock, given the surge in interest pertaining to the use of $\alpha_{2}$-adrenoceptor agonists in this setting $[2,3]$.

\section{Septic Shock}

2.1. Septic Shock. The definition of septic shock includes a systolic blood pressure (SBP) $<90 \mathrm{mmHg}$, after adequate fluid replacement (commonly $>30 \mathrm{~mL} \cdot \mathrm{kg}-1$ in $<6 \mathrm{~h}$ ) and the need for vasopressor drugs for more than $1 \mathrm{~h} \mathrm{[4]}$ or for $4 \mathrm{~h}$ (minimal requirements of NA $>0.05 \mu \mathrm{g} \cdot \mathrm{kg}-1 \cdot \mathrm{min}-1$ ). Earlier series have reported a death toll of $\approx 70 \%$ [5] and recent series still report a high mortality (27\% [6], 20\% [7], and $16 \%$ [8]). Refractory septic shock is defined as a requirement for NA $>0.25 \mu \mathrm{g} \cdot \mathrm{kg}-1 \cdot \mathrm{min}-1$ ( $>1 \mathrm{mg} \cdot \mathrm{h}-1 / 70 \mathrm{~kg}$ ) [9] or $>$ $0.5 \mu \mathrm{g} \cdot \mathrm{kg}-1 \cdot \mathrm{min}-1$ [10]. Other definitions are (a) worsening circulatory failure despite aggressive use of vasopressors and (b) increasing lactic acidosis despite $6 \mathrm{~h}$ of extrarenal replacement therapy (ERRT) [11]. In a study of 51 consecutive patients with septic shock [12], an overall $45 \%$ mortality was observed. Sixteen patients presented with refractory septic shock and death (31\% of the enrolled patients). The definition of refractory septic shock of this group [12] was no reversal of 
shock (i.e., an inability to sustain SBP $>90 \mathrm{mmHg}$ for $>24 \mathrm{~h}$ without NA):

(a) In the refractory septic shock group, the mortality over $48 \mathrm{~h}$ was $19 \%$, given the whole 51 patients: ten patients $(62 \%$ of the patients in refractory septic shock) died within $48 \mathrm{~h}$ of circulatory failure. The mortality over $28 \mathrm{~d}$ (early circulatory failure and late multiple organ failure) in the refractory septic shock patients was $100 \%$. The NA requirement was $\approx 2.6 \mu \mathrm{g}-$ $1 \cdot \mathrm{kg}-1 \cdot \mathrm{min}-1$, that is, $\approx 11 \mathrm{mg} \cdot \mathrm{h}-1$.

(b) In nonrefractory septic shock, the mortality over $28 \mathrm{~d}$ was $20 \%$. The NA requirements were $\approx 1 \mu \mathrm{g}-1 \cdot \mathrm{kg}$ $1 \cdot \mathrm{min}-1$, that is, $\approx 4 \mathrm{mg} \cdot \mathrm{h}-1$ [12].

2.2. Treatment. The initial treatment for septic shock is volume loading, but the adequacy of volume loading is poorly defined [1]. Presumably, the best index is the collapsibility of the vena cava (superior vena cava [13] or inferior vena cava) or absence of response to passive leg rising. Thus, adequacy of volume load is assessed when little or no change occurs in the diameter of the inferior or superior vena cava or when additional volume loading evokes no additional increase in cardiac output (CO). There is ongoing controversy regarding the balance between the necessity to achieve adequate volemia, during the first $24-72 \mathrm{~h}$, and the necessity to avoid increased lung water by normalizing the net weight gain, as early as possible.

The second line of therapy is the use of vasopressors, usually NA, to achieve a MAP $\geq 65 \mathrm{mmHg}$. The dose of NA required varies from $\approx 1 \mu \mathrm{g}-1 \cdot \mathrm{kg}-1 \cdot \mathrm{min}-1$ to $\approx 2.6 \mu \mathrm{g}-1 \cdot \mathrm{kg}-$ $1 \cdot$ min-1, respectively, in nonrefractory versus refractory septic shock (4 to $11 \mathrm{mg} \cdot \mathrm{h}-1$ ) [12]. However, the same established group [14] uses NA as high as 50-100 mg.h-1 to treat refractory septic shock. Secondly, setting the MAP $\geq 65 \mathrm{mmHg}$ may be arbitrary: $\mathrm{BP}$ is too low when dealing with patients with preexisting hypertension [15] or with low functional capillary density [16]. Conversely, BP is too high if MAP is the only parameter to be followed (i.e., disregarding the indices of global tissue perfusion such as trends in arterial lactate concentration, mixed venous $\mathrm{O}_{2}$ saturation or superior vena cava $\mathrm{O}_{2}$ saturation, and arterial-venous $\mathrm{CO}_{2}$ gradient). There is also a controversy regarding the time NA treatment is instituted. Most commonly, NA is administered early in sepsis, especially if diastolic BP is low, but early institution of vasopressor treatment before achieving adequate global perfusion is associated with worse outcome [17] suggesting that maldistribution of blood flow may be increased by the liberal use of vasopressor in the setting of septic shock [17]. Therefore, a three-step strategy has been proposed [18]. First, as soon as possible [19], restore volume and peripheral perfusion using iterative monitoring of global tissue perfusion. Second, administer NA to maintain MAP $\geq 50 \mathrm{mmHg}$ (lower limit of cerebral/coronary autoregulation in normal humans, with a higher MAP if coronary/cerebral perfusion is endangered). Third, optimize kidney perfusion (as an index of single organ perfusion), after adequate global perfusion, by increasing the dose of NA [18].

\section{Microcirculation}

3.1. Uncoupling between the Peripheral and Central Compartments. One of the key problems faced by the intensivist in the setting of septic shock is an "uncoupling" between the macrocirculation ("central compartment": brain, heart, and lung) and the microcirculation. Within this schema, central compartment versus microcirculation, the kidney presents with peculiarities: given the large volume of blood it receives per time unit, the kidney is part of the central circulation. On the other hand, the microcirculation of the kidney is disrupted by sepsis, as any other major central organ: the clinical answer lies in a urine output $>0.5 \mathrm{~mL} \cdot \mathrm{h}-1$ as an index of adequate microcirculation.

Tissue blood flow is driven by metabolic demand, not by blood pressure (BP). At rest, in the healthy volunteer, this implies that the capillaries are alternatively perfused and then not perfused. In turn, this implies, in the healthy volunteer, that the blood volume needed to operate the whole circulatory system is kept to a minimum because the active part of the circulatory system is also kept to a minimum. By contrast, during exercise, muscle blood flow, under sympathetic restraint, increases 100-fold [20] with maximal capillary perfusion attained in $15 \mathrm{~s}$, compatible with a metabolic demand, restrained by sympathetic activation. Diving mammals are able to store massive loads of lactate at the periphery during diving and recirculate this acid load very quickly, getting ready for the next dive within minutes [21]. Accordingly, elite long-distance runners handle severe lactic acidosis and recirculate this load quickly upon completion of run.

At variance with data gathered in the 1930 s, recent results argue against the existence of precapillary sphincters that would allow independent, active control of individual capillaries. Arterioles are enmeshed in a rich plexus of sympathetic nerves and electrical stimulation leads to vasoconstriction spreading along the whole arteriole [22]. The sympathetic nervous system is activated by pressure (cardiac and vasomotor sympathetic baroreflexes), $\mathrm{CO}_{2}-\mathrm{H}^{+}-\mathrm{O}_{2}$ (chemoreflexes), or metabolism (metaboreflex). The large proximal arteries are controlled primarily by stimulation of $\alpha_{1}$-adrenoceptors, whereas small distal arteries are controlled mainly by stimulation of $\alpha_{2}$-adrenoceptors [20]. There is evidence that constriction of microvessels mediated by $\alpha_{2}$-adrenoceptors may be more sensitive to acidosis, compared with those mediated by $\alpha_{1}$-adrenoceptors, but it is unclear if this leads to a better local control of terminal arterioles by metabolic demand [23]. Furthermore, it is unclear how this acidosisevoked vasodilatation of small arterioles relates to the microcirculatory dysfunction observed during septic shock and massive sympathoactivation. The untested implication is that prolonged tissue hypoxia, or prolonged unloading of arterial baroreceptors, leads to prolonged, metabolically mediated, sympathetic activation. In turn, is this sympathetic activation instrumental in perpetuating tissue hypoxia? Conversely, does sympathetic deactivation alleviate peripheral shunting?

What happens to microvascular flow in to septic shock? This is not crystal clear. However, there is evidence that, in skeletal muscle, there is a large heterogeneity in the flow 
rate in capillaries [24]. Given normal BP in a rat model of peritonitis (caecal ligation and perforation) [24], a decrease in continuous blood flow and of normal blood flow was observed, while an increase in stopped flow was observed. The proportion of fast to normal flow increased, possibly due to a convective arterial-venous shunt. The oxygen saturation is lower at the venular end of the capillaries. The increase in oxygen extraction $\left(\mathrm{O}_{2}\right.$ ER) was directly related to the extent of stopped flow ( 5 times the $\mathrm{O}_{2}$ ER observed in controls). This corresponded to a loss of $50 \%$ of perfused capillaries. Taken together, these data indicate a patchy and disperse maldistribution of $\mathrm{O}_{2}$ during sepsis, as opposed to an inability to utilize $\mathrm{O}_{2}$ [24], that is, a cytopathic hypoxia [25]. The authors conclude the following: (a) increasing the delivery of oxygen to supranormal levels may not improve tissue oxygenation if the increased $\mathrm{O}_{2}$ supply cannot be properly distributed and (b) early treatment aimed at restoring uniform distribution of $\mathrm{O}_{2}$...may lead to improve outcomes [24]. This summarizes the present challenge. The speculation is that some capillaries are vasodilated due to NO excess and thus need NO inhibition. By contrast, flow is stopped in a large proportion of the capillaries: do these stopped capillaries need NO donors? All together this makes the systemic administration of NO inhibitors versus donors a challenge.

In septic humans, a reduced density of perfused sublingual capillaries is observed in nonsurvivors [26], irrespective of a similar circulatory and oxygenation profile observed in survivors versus nonsurvivors. Survival is associated with the increase in small vessel perfusion over the first $24 \mathrm{~h}$ but not associated with the overall circulatory and oxygenation variables [27]. Furthermore, there is a strong association between the delay in beginning therapy and outcome, compatible with extensive microcirculatory defects and their consequences, that is, multiple organ failure [19]. Volume load improves microcirculation during early but not late sepsis [28], suggestive of damage to the microcirculation. Additionally, the first bolus of volume loading improves all the indices of microcirculation, with no further improvement with a second bolus [29]: does this imply minimizing volume load during septic shock based on a microcirculatory index? The proportion of perfused vessels is unrelated to the administration of vasopressors [26]. A weak but significant correlation exists between small vessel perfusion, increasing $\mathrm{pH}$ and decreasing arterial lactate levels [26], which does not necessarily imply causality. When NA was used to increase BP from 65 to $85 \mathrm{mmHg}$, the largest increase in perfused capillary density was observed in patients presenting with the lowest perfused capillary density, suggestive of a possible effect of BP on functional capillary density: do the sicker patients need a higher BP? By contrast, the patients with the highest baseline perfused capillary density showed a reduction in perfused capillary density [16]. This suggests that individualized titration of NA based on the state of the microcirculation may be beneficial.

Finally, no correlation was observed between the slope of recovery to thenar muscle ischemia and NA requirements [30], although a weak correlation was observed between NA requirement and recovery during ischemia of the thenar muscle [12]. Therefore, NA requirement and the extent of microcirculatory defects are poorly related: "the alterations in thenar $\mathrm{O}_{2}$ saturation... are more related to the sepsis...itself and its severity than to mean arterial pressure and the dose of vasopressor agents" [30].

\section{Pressor Response to Noradrenaline}

Reduced pressor responsive to NA is a major challenge for clinicians treating septic patients. The effects of a number of treatments have been studied to determine if they improve the reduced pressor responsiveness to NA in sepsis.

4.1. Nitric Oxide Inhibitors. Based on the assumption of generalized nitric oxide (NO) excess in sepsis and subsequent excessive vasodilation, $\mathrm{NO}$ inhibitors have been tested [31]. Briefly (a) in septic patients, NO synthase (NOS) inhibitors (N-monomethyl-L-arginine: L NNMA) increased BP and lowered CO in a dose-dependent manner [32,33], with a $40 \%$ reduction in NA requirements [34], and (b) the changes evoked by L NNMA (inhibition of NO synthase) were reversed by L arginine [33]. However, a large study was stopped because of increased mortality in the group treated with a NOS inhibitor [35]. Studies in an ovine model of hyperdynamic septic shock showed that nonselective NOS inhibition restored BP, but not renal function, and a selective inhibitor of inducible NOS had no effect on BP or renal function $[36,37]$. Therefore, NOS inhibitors do not restore, in septic shock, the delicate tuning between active, perfused capillaries and inactive, unperfused capillaries governed by local metabolic demand in the resting healthy volunteer.

Another NO inhibitor, methylene blue (MB), improved the circulatory profile (increased stroke volume and reduced tachycardia) and reduced the NA requirements by $87 \%$, as early as one hour after beginning of administration [38, 39]. A meta-analysis favored the use of $M B$ in hypotensive patients, including septic shock patients (mortality: MB: $16 \%$; control: 23\%) [40]. To our knowledge, no further large-scale randomized study has taken up the issue.

4.2. NO Donors. In a nonrandomized study, the NO donor nitroglycerin (NTG) was administered during septic shock (bolus: $0.5 \mathrm{mg}$; continuous administration: $0.5-4.0 \mathrm{mg} \cdot \mathrm{h}-1$ ), after volume load (central venous pressure $>12 \mathrm{mmHg}$ ) and vasopressor administration. A major improvement in the microcirculation was observed, with survival in 7 out of 8 patients [41]. A similar response has also been observed with a NTG patch (12-18 mg every $4 \mathrm{~h}$ ) [42] and in a randomized study there was evidence that NTG improved perfusion of small vessels in septic patients [43]. Furthermore, the trend in lactate concentrations improved in the NTG group. The study was not conclusive regarding whether NTG reduced the length of stay in the critical care unit, but there was a higher mortality in the NTG group [43]. However, the small size sample and the inclusion of septic patients together with septic shock patients does not allow one to reach a definitive conclusion. 
4.3. Hydrocortisone (HSHC). Low dose steroids generated little increase in BP in septic shock patients receiving or not receiving phenylephrine, except when very high doses of phenylephrine were used [44]. HSHC hastened the reversal of septic shock (HSHC: 3.3 days versus placebo; 5.8 days in patients in whom shock was reversed; 76 and $70 \%$ in the HSHC and placebo groups, resp.), irrespective of a positive or negative response to corticotropin. However, mortality was unchanged, irrespective of group. A higher incidence of new episodes of sepsis or septic shock was observed in the HSHC group.

4.4. $\alpha_{2}$-Adrenoceptor Agonists. As the drugs cited above were not overwhelmingly successful in treating sepsis, our group has examined a novel and counterintuitive approach: the use of $\alpha_{2}$-adrenoceptor agonists. In two cases [45], treatment with the $\alpha_{2}$-adrenoceptor agonist, clonidine $(1 \mu \mathrm{g} \cdot \mathrm{kg}-1 \cdot \mathrm{h}-1)$, in addition to state-of-the-art treatment, reduced NA requirements in (a) a patient presenting with HIV and terminal pulmonary sepsis (-45\%) [45] and (b) a neonate presenting with necrotizing enterocolitis ( $-90 \%$, submitted). In addition, we have documented this reduction in requirement for NA in rat [46] and sheep [47] experimental models of sepsis, using high and low doses, respectively, of the $\alpha_{2}$-adrenoceptor agonists, clonidine and dexmedetomidine. Furthermore, the pressor responsiveness to a noncatecholaminergic vasopressor, angiotensin II, was also reduced by clonidine treatment [47].

One possible mechanism [48] for this effect of $\alpha_{2}$ adrenoceptor agonists in sepsis is that, during septic shock, as during exercise [49], there is increased sympathetic nerve activity and endogenous plasma catecholamines [50-52] with a downregulation in responsiveness to stimulation of $\alpha_{1}$ - and $\beta$-adrenoceptors, which may result from reduced binding or reduced sensitivity/intracellular coupling. Conversely, the other side of this working hypothesis [48] is that, during rest after exercise, or after lowering plasma catecholamine concentrations with either pharmacologically evoked $\alpha_{2}$ adrenoceptor agonists or those occurring spontaneously during recovery from sepsis, the downregulation of $\alpha_{1}$-adrenoceptors is converted to upregulation, with an increased pressor response to vasopressors.

Clonidine reduces sympathetic nerve activity to the heart and vasculature by a direct central action, which is its main mechanism of action as an antihypertensive drug [53, 54]. How can this central action of clonidine to reduce BP in hypertensive patients be reconciled with an increased pressor response and lowered NA requirement in patients with sepsis? A recent experimental study indicates that treatment with clonidine reduced renal sympathetic nerve activity from high to normal levels [47]. Together with reductions in sympathetic nerve activity to other organs, this is likely associated with a decrease in plasma catecholamines concentrations and is compatible with our working hypothesis. It remains to be determined whether the maldistribution of capillary perfusion in sepsis [24] is improved by treatment with $\alpha_{2}$-adrenoceptor agonists and if so whether this is due to its central sympathetic deactivation or to a direct vascular action.
Given our reports that clonidine reduced the requirement for NA in sepsis [45] and our demonstrations of improved pressor responsiveness in small [46] and large [47] animal models of sepsis, it is essential that evidence-based documentation of the effects of $\alpha_{2}$-adrenoceptor agonists in human septic shock is obtained. A concern may be the possible harm to the patient by using an antihypertensive agent during septic shock, indeed a bold and counterintuitive move. The answer appears three-fold. First, adequate volume loading before administration of the $\alpha_{2}$-adrenoceptor agonist is needed. As the microcirculation corrects slowly (as shown by the changes in arterial lactate, central $\mathrm{O}_{2}$ saturation, and arterial to venous $\mathrm{CO}_{2}$ gradient) the most expeditious way would be to optimize the central compartment: little or no collapsibility of the inferior or superior vena cava during ventilation would guarantee no more increase in $\mathrm{CO}$ or little response to passive leg rising. Second, the definition of an adequate BP is needed: permissive hypotension [18] (MAP $\geq 50 \mathrm{mmHg}$ ) versus standard MAP $\geq 65 \mathrm{mmHg}$ [1] versus higher MAP in selected patients $[15,16]$. Third, given the very high circulatory-related mortality in refractory septic shock [12], the patients in this category may be administered with a "compassionate" treatment under the Helsinki Declaration ("where proven prophylactic, diagnostic, and therapeutic methods do not exist or have been ineffective, the physician, with informed consent from the patient, must be free to use unproven or new prophylactic, diagnostic, and therapeutic measures, if in the physician's judgment it offers hope of saving life, reestablishing health, or alleviating suffering; where possible, these measures should be made the object of research, designed to evaluate their safety and efficacy"). The primary end-point will be increased pressor responsiveness to vasopressors. Such a clinical trial should also address whether there are improvements in the microcirculation. For, example, does sympathetic deactivation with an $\alpha_{2}$ adrenoceptor agonist reverse the peripheral microcirculatory shut-down and reduce inflammation and multiple organ failure? An end-point on mortality would require a large sample, not compatible with a preliminary trial.

4.5. Clonidine versus Dexmedetomidine. Which alpha-2 agonist is to be selected to head into a preliminary clinical trial? Clonidine has 2 disadvantages and one advantage: (a) a slow onset (3-6h) when administered slowly and intravenously to evoke no precipitous sympathetic deactivation. In the context of inadequate volemia ( $\$$ treatment), precipitous sympathodeactivation will lead to a precipitous fall in BP. $A$ rigorous proviso should be made to address the issue of an optimized volemia before the initiation of sympathetic deactivation. When opposed to clonidine, dexmedetomidine will be the drug of choice as its sedative effect is observed after 30-60 min. However, the issue in the setting of septic shock is not to observe a fast onset for sedation, but to observe a putative, increased pressor response to NA, without precipitous fall in BP. As the patient presenting with septic shock is to stay in the CCU for an extended period of time, a faster onset of sedative versus pressor effect will make little pharmacoeconomic difference. Our observations [45] 
show that clonidine increases the pressor response to NA within 2-3 h. Nevertheless, a comparison will be needed to address the superiority of any of the two clinically available alpha-2 agonists (b) in healthy volunteers, a long elimination half-life of clonidine (circa 24 h) [55] as opposed to a short elimination half-life for dexmedetomidine (circa $3 \mathrm{~h}$ ). Any untoward effect will presumably last longer with clonidine. This is not the issue: the point is how to get increased pressor response to NA without inducing a major fall in BP during the initial administration of the alpha-2 agonist, and not the possible length of time of such an exaggerated fall. The answer rests with adequate volemia before heading to sympathetic deactivation ( $\$$ treatment). (c) Clonidine is eliminated via the kidney as opposed to dexmedetomidine eliminated via the liver. Many patients presenting with septic shock require ERRT. Thus, any overdose of clonidine will be easily eliminated. By contrast, dexmedetomidine may not generate an overdose secondary to kidney failure, easing the management. However, administration of dexmedetomidine may become tricky if the patient presents liver failure, as extra-liver replacement therapy is no widely available. Lastly, the key point is the dose of alpha-2 agonist needed to generate sympathetic deactivation, thus increased pressor response: the dose of clonidine we used [45] (1 mcg.kg-1.h-1) needs to be refined to achieve maximal sympathodeactivation with minimal side effects.

\section{Conclusion}

In healthy volunteers, the microcirculation is constantly shunting blood away from inactive to active territories and vice versa. This fine tuning allows the whole body to be adequately perfused with a blood volume of only $5 \mathrm{~L}$, even in the setting of strenuous exercise. By contrast, in the setting of septic shock, the human organism apparently needs a higher blood volume (or at least reestablishment of adequate blood volume) and a recoupling of the microcirculation with the central compartment. At present, physicians are unable to emulate what humans achieve after long-distance running or diving mammals when they reach the surface, that is, reorganizing a shut-down microcirculation to force $\mathrm{O}_{2}$ through capillaries and generate a quick wash-out of anaerobic metabolites.

A different issue is the pressor response to NA, which defines, when completely blunted, refractory septic shock. Steroids increase the response to phenylephrine, but only when very high doses of phenylephrine are used [44]. NO inhibitors have been withdrawn from trial based on sideeffects, possibly related to the dose of drug. Methylene blue has not been assessed in a large double blind trial to handle refractory hypotension in the setting of septic shock. In our studies of $\alpha_{2}$-adrenoceptor agonists, we have observed a large (45-90\%) reduction in NA requirements in terminal septic shock [45] and in necrotizing enterocolitis. Simultaneously, in our patients [45], peripheral mottling vanished over hours: this suggests that the microcirculation may have been progressively recoupled to the central compartment. We replicated an increase in pressor responsiveness to NA with dexmedetomidine and clonidine in the setting of mild sepsis in rat [46] and sheep [47]. The working hypothesis [45, $48]$ is that $\alpha_{2}$-adrenoceptor agonist mediated sympathetic deactivation lowers the release of endogenous NA, allowing upregulation of vascular $\alpha_{1}$-adrenoceptors back towards normal levels. This hypothesis $[45,48]$ is to be put to the acid test in the setting of human septic shock, preferably refractory. Again, a rigorous proviso should be made to address the issue of an optimized volemia before the initiation of sympathetic deactivation.
Abbreviations
BP: Blood pressure
CO: Cardiac output
ERRT: Extrarenal replacement therapy
HSHC: Hemisuccinate of hydrocortisone
MAP: Mean arterial pressure
MB: Methylene blue
NA: Noradrenaline
NO: Nitric oxide
NOS: NO synthase
NTG: Nitroglycerin
O2ER: Oxygen extraction ratio
SBP: $\quad$ Systolic blood pressure.

\section{Conflict of Interests}

L. Quintin holds a US patent (8 846606 B2, September 30, 2014) on method and drug composition for treating septic shock hypotension. The other authors declare no conflict of interests.

\section{References}

[1] R. P. Dellinger, M. M. Levy, A. Rhodes et al., "Surviving sepsis campaign: international guidelines for management of severe sepsis and septic shock, 2012," Intensive Care Medicine, vol. 39, no. 2, pp. 165-228, 2013.

[2] M. W. Dünser and I. Gradwohl-Matis, " $\alpha 2$-agonists to restore adrenergic vasoconstrictor responsiveness in septic shock: thinking outside of the box or fishing in the wrong pond?" Critical Care Medicine, vol. 41, no. 12, pp. 2838-2840, 2013.

[3] I. Gradwohl-Matis and M. W. Dünser, "Reverse physiology: applying an antihypertensive drug to increase arterial blood pressure in septic shock," Critical Care Medicine, vol. 43, no. 7, pp. 1548-1550, 2015.

[4] C. L. Sprung, D. Annane, D. Keh et al., "Hydrocortisone therapy for patients with septic shock," The New England Journal of Medicine, vol. 358, no. 2, pp. 111-124, 2008.

[5] J. A. Russell, "Management of sepsis," The New England Journal of Medicine, vol. 355, no. 16, pp. 1699-1713, 2006.

[6] J.-Y. Lefrant, L. Muller, A. Raillard et al., "Reduction of the severe sepsis or septic shock associated mortality by reinforcement of the recommendations bundle: a multicenter study," Annales Francaises d'Anesthesie et de Reanimation, vol. 29, no. 9, pp. 621-628, 2010.

[7] D. Talmor, D. Greenberg, M. D. Howell, A. Lisbon, V. Novack, and N. Shapiro, "The costs and cost-effectiveness of an integrated sepsis treatment protocol," Critical Care Medicine, vol. 36, no. 4, pp. 1168-1174, 2008. 
[8] M. Zambon, M. Ceola, R. Almeida-de-Castro, A. Gullo, and J.L. Vincent, "Implementation of the Surviving Sepsis Campaign guidelines for severe sepsis and septic shock: we could go faster," Journal of Critical Care, vol. 23, no. 4, pp. 455-460, 2008.

[9] P. D. Annane, P. E. Bellissant, and J.-M. Cavaillon, "Septic shock," The Lancet, vol. 365, no. 9453, pp. 63-78, 2005.

[10] K. Donadello and F. S. Taccone, "Refractory septic shock: who and how should we purify?" Minerva Anestesiologica, vol. 81, no. 5, pp. 475-477, 2015.

[11] A. Vieillard-Baron, V. Caille, C. Charron et al., "Reversal of refractory septic shock with drotrecogin alpha (activated)," Intensive Care Medicine, vol. 35, no. 7, pp. 1204-1209, 2009.

[12] M. Conrad, P. Perez, C. Thivilier, and B. Levy, "Early prediction of norepinephrine dependency and refractory septic shock with a multimodal approach of vascular failure," Journal of Critical Care, vol. 30, no. 4, pp. 739-743, 2015.

[13] A. Vieillard-Baron, K. Chergui, A. Rabiller et al., "Superior vena caval collapsibility as a gauge of volume status in ventilated septic patients," Intensive Care Medicine, vol. 30, no. 9, pp. 17341739, 2004.

[14] S. Collin and B. Levy, "Pathophysiological mechanisms of vascular hyporesponsiveness in shock states," Reanimation, vol. 17, no. 2, pp. 101-110, 2008.

[15] P. Asfar, F. Meziani, J.-F. Hamel et al., "High versus low bloodpressure target in patients with septic shock," The New England Journal of Medicine, vol. 370, no. 17, pp. 1583-1593, 2014.

[16] A. Dubin, M. O. Pozo, C. A. Casabella et al., "Increasing arterial blood pressure with norepinephrine does not improve microcirculatory blood flow: a prospective study," Critical Care, vol. 13, article R92, 2009.

[17] S. Subramanian, M. Yilmaz, A. Rehman, R. D. Hubmayr, B. Afessa, and O. Gajic, "Liberal vs. conservative vasopressor use to maintain mean arterial blood pressure during resuscitation of septic shock: an observational study," Intensive Care Medicine, vol. 34, no. 1, pp. 157-162, 2008.

[18] M. W. Dünser, J. Takala, A. Brunauer, and J. Bakker, "Re-thinking resuscitation: leaving blood pressure cosmetics behind and moving forward to permissive hypotension and a tissue perfusion-based approach," Critical Care, vol. 17, article 326, 2013.

[19] O. Abid, S. Akça, P. Haji-Michael, and J.-L. Vincent, "Strong vasopressor support may be futile in the intensive care unit patient with multiple organ failure," Critical Care Medicine, vol. 28, no. 4, pp. 947-949, 2000.

[20] S. S. Segal, "Regulation of blood flow in the microcirculation," Microcirculation, vol. 12, no. 1, pp. 33-45, 2005.

[21] J. F. Nunn, "Respiratory aspects of high pressure and diving," in Applied Respiratory Physiology, pp. 321-331, Butterworths, London, UK, 1987.

[22] J. E. Hungerford, W. C. Sessa, and S. S. Segal, "Vasomotor control in arterioles of the mouse cremaster muscle," The FASEB Journal, vol. 14, no. 1, pp. 197-207, 2000.

[23] K. M. McGillivray-Anderson and J. E. Faber, "Effect of acidosis on contraction of microvascular smooth muscle by $\alpha 1$ - and $\alpha 2$ adrenoceptors. implications for neural and metabolic regulation," Circulation Research, vol. 66, no. 6, pp. 1643-1657, 1990.

[24] C. G. Ellis, R. M. Bateman, M. D. Sharpe, W. J. Sibbald, and R. Gill, "Effect of a maldistribution of microvascular blood flow on capillary $\mathrm{O}_{2}$ extraction in sepsis," American Journal of Physiology-Heart and Circulatory Physiology, vol. 282, no. 1, pp. H156-H164, 2002.
[25] M. P. Fink, "Bench-to-bedside review: cytopathic hypoxia," Critical Care, vol. 6, no. 6, pp. 491-499, 2002.

[26] D. De Backer, J. Creteur, J.-C. Preiser, M.-J. Dubois, and J.-L. Vincent, "Microvascular blood flow is altered in patients with sepsis," American Journal of Respiratory and Critical Care Medicine, vol. 166, no. 1, pp. 98-104, 2002.

[27] Y. Sakr, M.-J. Dubois, D. De Backer, J. Creteur, and J.-L. Vincent, "Persistent-microcirculatory alterations are associated with organ failure and death in patients with septic shock," Critical Care Medicine, vol. 32, no. 9, pp. 1825-1831, 2004.

[28] G. Ospina-Tascon, A. P. Neves, G. Occhipinti et al., "Effects of fluids on microvascular perfusion in patients with severe sepsis," Intensive Care Medicine, vol. 36, no. 6, pp. 949-955, 2010.

[29] J. Pottecher, S. Deruddre, J.-L. Teboul et al., "Both passive leg raising and intravascular volume expansion improve sublingual microcirculatory perfusion in severe sepsis and septic shock patients," Intensive Care Medicine, vol. 36, no. 11, pp. 1867-1874, 2010.

[30] J. Creteur, T. Carollo, G. Soldati, G. Buchele, D. De Backer, and J.-L. Vincent, "The prognostic value of muscle $\mathrm{StO}_{2}$ in septic patients," Intensive Care Medicine, vol. 33, no. 9, pp. 1549-1556, 2007.

[31] J.-L. Vincent, H. Zhang, C. Szabo, and J.-C. Preiser, "Effects of nitric oxide in septic shock," American Journal of Respiratory and Critical Care Medicine, vol. 161, no. 6, pp. 1781-1785, 2000.

[32] A. Petros, G. Lamb, A. Leone, S. Moncada, D. Bennett, and P. Vallance, "Effects of a nitric oxide synthase inhibitor in humans with septic shock," Cardiovascular Research, vol. 28, no. 1, pp. 34-39, 1994.

[33] J. A. Lorente, L. Landin, R. De Pablo, E. Renes, and D. Liste, "L-arginine pathway in the sepsis syndrome," Critical Care Medicine, vol. 21, no. 9, pp. 1287-1295, 1993.

[34] J. A. M. Avontuur, R. P. Tutein Nolthenius, J. W. van Bodegom, and H. A. Bruining, "Prolonged inhibition of nitric oxide synthesis in severe septic shock: a clinical study," Critical Care Medicine, vol. 26, no. 4, pp. 660-667, 1998.

[35] A. López, J. A. Lorente, J. Steingrub et al., "Multiple-center, randomized, placebo-controlled, double-blind study of the nitric oxide synthase inhibitor 546C88: effect on survival in patients with septic shock," Critical Care Medicine, vol. 32, no. 1, pp. 2130, 2004.

[36] K. Ishikawa, R. Bellomo, and C. N. May, "The impact of intrarenal nitric oxide synthase inhibition on renal blood flow and function in mild and severe hyperdynamic sepsis," Critical Care Medicine, vol. 39, no. 4, pp. 770-776, 2011.

[37] K. Ishikawa, P. Calzavacca, R. Bellomo, M. Bailey, and C. N. May, "Effect of selective inhibition of renal inducible nitric oxide synthase on renal blood flow and function in experimental hyperdynamic sepsis," Critical Care Medicine, vol. 40, no. 8, pp. 2368-2375, 2012.

[38] F. Schneider, P. Lutun, M. Hasselmann, J. C. Stoclet, and J. D. Tempé, "Methylene blue increases systemic vascular resistance in human septic shock. Preliminary observations," Intensive Care Medicine, vol. 18, no. 5, pp. 309-311, 1992.

[39] M. Y. Kirov, O. V. Evgenov, N. V. Evgenov et al., "Infusion of methylene blue in human septic shock: a pilot, randomized, controlled study," Critical Care Medicine, vol. 29, no. 10, pp. 1860-1867, 2001.

[40] L. Pasin, M. Umbrello, T. Greco et al., "Methylene blue as a vasopressor: a meta-analysis of randomised trials," Critical Care and Resuscitation, vol. 15, no. 1, pp. 42-48, 2013. 
[41] P. E. Spronk, C. Ince, M. J. Gardien, K. R. Mathura, H. M. O.V. Straaten, and D. F. Zandstra, "Nitroglycerin in septic shock after intravascular volume resuscitation," The Lancet, vol. 360, no. 9343, pp. 1395-1396, 2002.

[42] F. B. Cerra, J. Hassett, and J. H. Siegel, "Vasodilator therapy in clinical sepsis with low output syndrome," Journal of Surgical Research, vol. 25, no. 2, pp. 180-183, 1978.

[43] E. C. Boerma, M. Koopmans, A. Konijn et al., "Effects of nitroglycerin on sublingual microcirculatory blood flow in patients with severe sepsis/septic shock after a strict resuscitation protocol: a double-blind randomized placebo controlled trial," Critical Care Medicine, vol. 38, no. 1, pp. 93-100, 2010.

[44] E. Bellissant and D. Annane, "Effect of hydrocortisone on phenylephrine-mean arterial pressure dose-response relationship in septic shock," Clinical Pharmacology and Therapeutics, vol. 68, no. 3, pp. 293-303, 2000.

[45] C. Pichot, P. Mathern, F. Khettab, M. Ghignone, A. Geloen, and L. Quintin, "Increased pressor response to noradrenaline during septic shock following clonidine?" Anaesthesia and Intensive Care, vol. 38, no. 4, pp. 784-785, 2010.

[46] A. Geloen, K. Chapelier, A. Cividjian et al., "Clonidine and dexmedetomidine increase the pressor response to norepinephrine in experimental sepsis: a pilot study," Critical Care Medicine, vol. 41, no. 12, pp. e431-e438, 2013.

[47] Y. R. Lankadeva, L. C. Booth, J. Kosaka et al., "Clonidine restores pressor responsiveness to phenylephrine and angiotensin II in ovine sepsis," Critical Care Medicine, vol. 43, no. 7, pp. e221-e229, 2015.

[48] C. Pichot, A. Géloën, M. Ghignone, and L. Quintin, "Alpha-2 agonists to reduce vasopressor requirements in septic shock?" Medical Hypotheses, vol. 75, no. 6, pp. 652-656, 2010.

[49] J. Butler, J. G. Kelly, K. O’Malley, and F. Pidgeon, “ $\beta$-adrenoceptor adaptation to acute exercise," The Journal of Physiology, vol. 344, no. 1, pp. 113-117, 1983.

[50] C. R. Benedict and J. A. Rose, "Arterial norepinephrine changes in patients with septic shock," Circulatory Shock, vol. 38, no. 3, pp. 165-172, 1992.

[51] R. Ramchandra, L. Wan, S. G. Hood, R. Frithiof, R. Bellomo, and C. N. May, "Septic shock induces distinct changes in sympathetic nerve activity to the heart and kidney in conscious sheep," The American Journal of Physiology-Regulatory Integrative and Comparative Physiology, vol. 297, no. 5, pp. R1247-R1253, 2009.

[52] M. W. Dünser and W. R. Hasibeder, "Sympathetic overstimulation during critical illness: adverse effects of adrenergic stress," Journal of Intensive Care Medicine, vol. 24, no. 5, pp. 293-316, 2009.

[53] M.-K. Sun and P. G. Guyenet, "Effect of clonidine and $\gamma$-aminobutyric acid on the discharges of medullo-spinal sympathoexcitatory neurons in the rat," Brain Research, vol. 368, no. 1, pp. 1-17, 1986.

[54] C. Mermet and L. Quintin, "Effect of clonidine on catechol metabolism in the rostral ventrolateral medulla: an in vivo electrochemical study," European Journal of Pharmacology, vol. 204, no. 1, pp. 105-107, 1991.

[55] D. Arndts, "New aspects of the clinical pharmacology of clonidine," Chest, vol. 83, no. 2, pp. 397-400, 1983. 


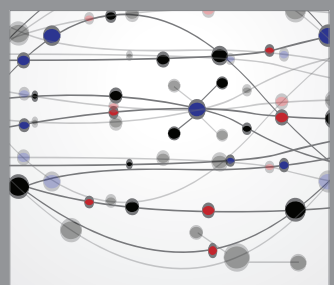

The Scientific World Journal
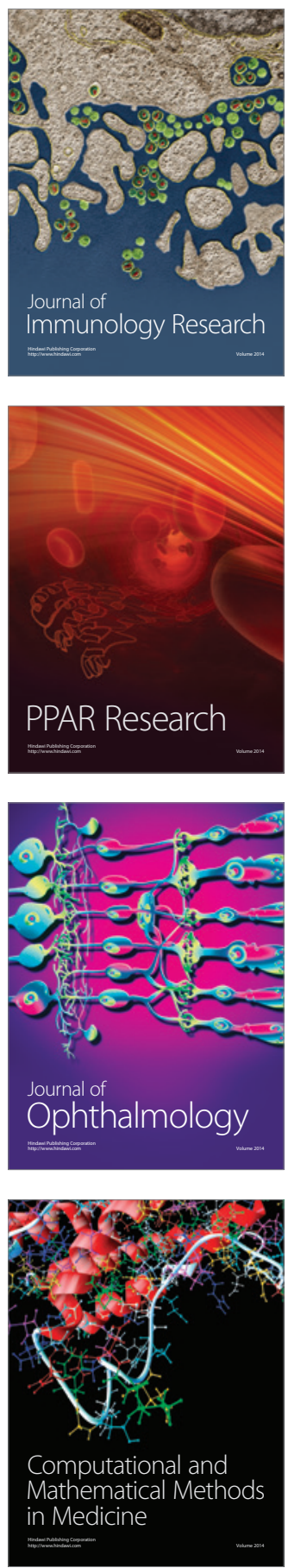

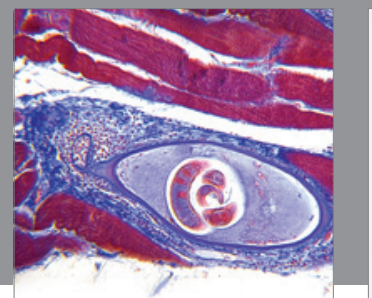

Gastroenterology

Research and Practice
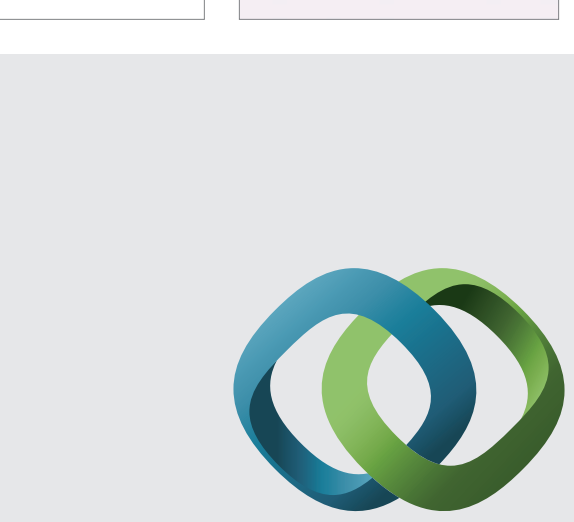

\section{Hindawi}

Submit your manuscripts at

http://www.hindawi.com
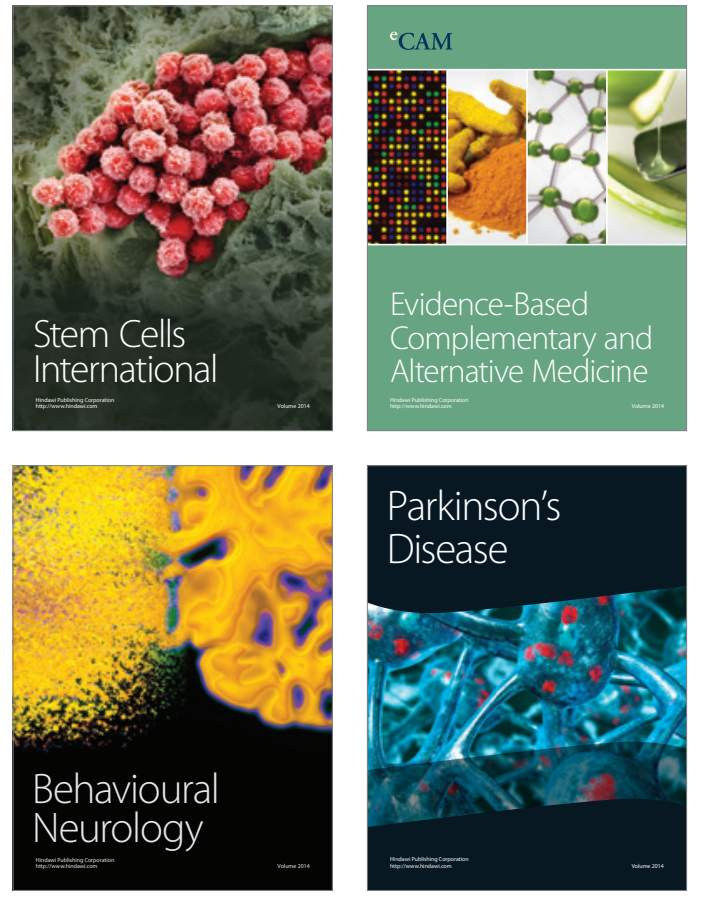
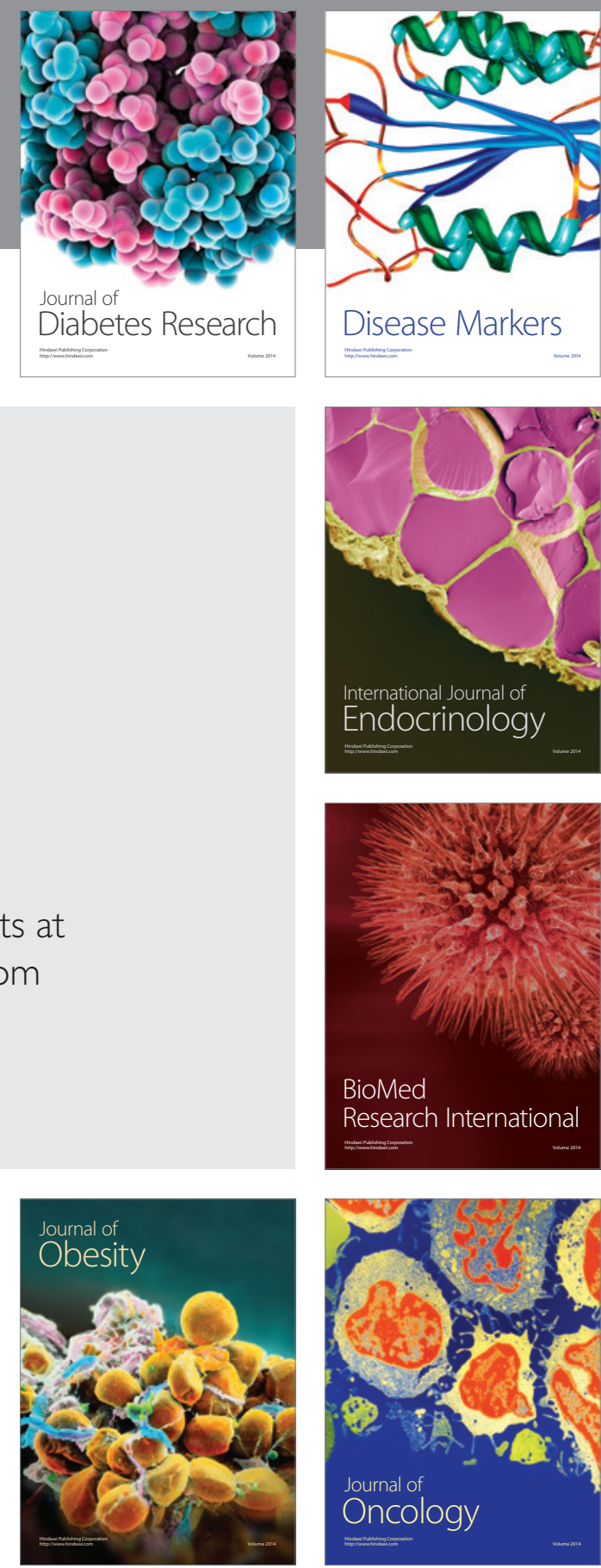

Disease Markers
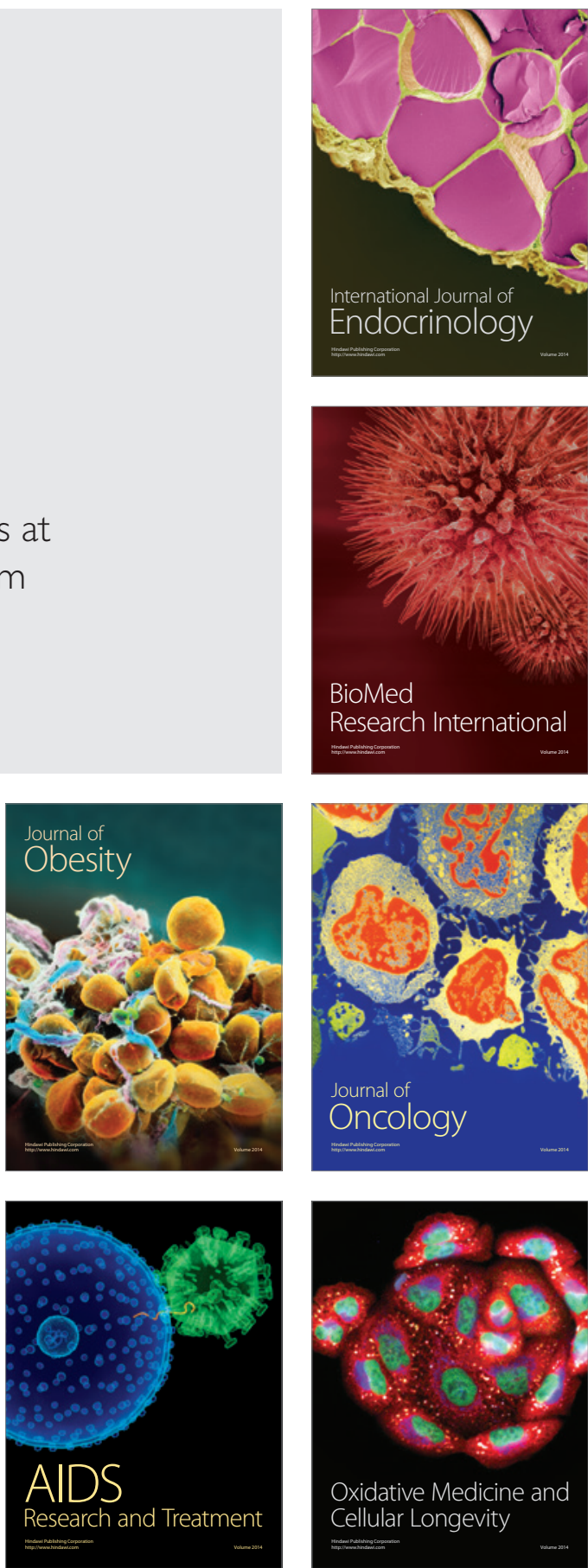